\title{
Peroxicretion: a novel secretion pathway in the eukaryotic cell
} Cees MJ Sagt*1, Peter J ten Haaft ${ }^{1}$, Ingeborg M Minneboo ${ }^{1}$, Miranda P Hartog1, Robbert A Damveld1, Jan Metske van der Laan1, Michiel Akeroyd ${ }^{1}$, Thibaut J Wenzel ${ }^{1}$, Francisca A Luesken², Marten Veenhuis ${ }^{3}$, Ida van der Klei ${ }^{3}$ and Johannes H de Winde ${ }^{1,4}$

Address: ${ }^{1}$ DSM Biotechnology Center, Beijerinck Laboratory, PO Box 1, 2600MA Delft, the Netherlands, ${ }^{2}$ Department of Microbiology Radboud University Nijmegen Toernooiveld 1, 6525ED Nijmegen, the Netherlands, ${ }^{3}$ Groningen University department of microbiology, Groningen, the Netherlands and ${ }^{4}$ Kluyver Centre for Genomics of Industrial Fermentation, Delft University of Technology, Department for Biotechnology, Julianalaan 67, 2628BC Delft, the Netherlands

Email: Cees MJ Sagt* - cees.sagt@dsm.com; Peter J ten Haaft - Peter.Haaft-ten@dsm.com; Ingeborg M Minneboo - i.minneboo@nedalco.nl; Miranda P Hartog - Miranda.hartog@dsm.com; Robbert A Damveld - Robbert.Damveld@dsm.com; Jan Metske van der Laan - Jan-Metske.Laanvan-der@dsm.com; Michiel Akeroyd - Michiel.Akeroyd@dsm.com; Thibaut J Wenzel - Thibaut.Wenzel@dsm.com;

Francisca A Luesken - f.luesken@science.ru.nl; Marten Veenhuis - m.veenhuis@rug.nl; Ida van der Klei - i.j.van.der.klei@rug.nl; Johannes H de Winde - J.H.deWinde@tudelft.nl

* Corresponding author

Published: 20 May 2009

BMC Biotechnology 2009, 9:48 doi:10.1 | 86//472-6750-9-48
Received: 28 April 2009

Accepted: 20 May 2009

This article is available from: http://www.biomedcentral.com/I472-6750/9/48

(C) 2009 Sagt et al; licensee BioMed Central Ltd.

This is an Open Access article distributed under the terms of the Creative Commons Attribution License (http://creativecommons.org/licenses/by/2.0), which permits unrestricted use, distribution, and reproduction in any medium, provided the original work is properly cited.

\begin{abstract}
Background: Enzyme production in microbial cells has been limited to secreted enzymes or intracellular enzymes followed by expensive down stream processing. Extracellular enzymes consists mainly of hydrolases while intracellular enzymes exhibit a much broader diversity. If these intracellular enzymes could be secreted by the cell the potential of industrial applications of enzymes would be enlarged. Therefore a novel secretion pathway for intracellular proteins was developed, using peroxisomes as secretion vesicles.
\end{abstract}

Results: Peroxisomes were decorated with a Golgi derived v-SNARE using a peroxisomal membrane protein as an anchor. This allowed the peroxisomes to fuse with the plasma membrane. Intracellular proteins were transported into the peroxisomes by adding a peroxisomal import signal (SKL tag). The proteins which were imported in the peroxisomes, were released into the extracellular space through this artificial secretion pathway which was designated peroxicretion. This concept was supported by electron microscopy studies.

Conclusion: Our results demonstrate that it is possible to reroute the intracellular trafficking of vesicles by changing the localisation of SNARE molecules, this approach can be used in in vivo biological studies to clarify the different control mechanisms regulating intracellular membrane trafficking. In addition we demonstrate peroxicretion of a diverse set of intracellular proteins. Therefore, we anticipate that the concept of peroxicretion may revolutionize the production of intracellular proteins from fungi and other microbial cells, as well as from mammalian cells. 


\section{Background}

The specificity of intracellular membrane trafficking is determined by multiple layers of control mechanisms that ensure that only appropriate organelles fuse with specific target compartments. These include Rab-GTPases [1] operating in conjunction with polyphosphoinositides [2] and Rab effectors [3] that frequently include multiprotein complexes. In eukaryotes, membrane fusion of secretory vesicles is mediated by SNARE-proteins [4] and specificity of membrane fusion is obtained by specific SNARE-protein interactions. In yeast, fusion of post-Golgi trafficking vesicles requires at least 10 genes including the RabGTPase Sec4 [5] the Exocyst multiprotein complex [6] and the SNAREs Snc1/2 [7] on the transport vesicle and Sso1/ $2[8]$ and Sec9 [9] on the plasma membrane. Moreover organelles can only fuse with target membranes once they are transported into close proximity, involving directed transport along cytoskeletal tracts [10]. The formation of the resulting SNARE-pin subsequently triggers membrane fusion [11]. The ER supplies the secretory route with membrane enclosed vesicles which travel from the ER via the Golgi towards the cell membrane.

The ER is very different from the cytosol regarding posttranslational protein modifications. $\mathrm{N}$-glycosylation of proteins in the ER is important for folding, degradation and quality control [12]. The cytosol does not contain an N-glycosylation machinery and as a consequence, soluble cytosolic proteins are not $\mathrm{N}$-glycosylated [13]. In addition, the reducing environment of the cytosol is very different from that in the ER and Golgi, where oxidizing conditions and specialized folding enzymes like Pdi1 and Ero1 facilitate disulfide bridge formation [14]. These fundamental differences between secretory pathway and cytosol complicate the routing of cytosolic proteins through the secretory pathway to yield active, secreted enzymes. In fact, literature does not describe any successful extracellular production of an intracellular protein through the secretory pathway. Cytosolic proteins preferentially fold into their active conformation with the aid of specific chaperones and folding enzymes, under the reducing conditions which are normal to the cytosol [15]. Recently it has been described that peroxisomes also may have their origin in the ER [16]. However they do not fuse with other compartments and SNARE molecules have not been detected on peroxisomes [17]. The peroxisome has all the necessary features to enable import of completely folded and mature intracellular proteins [18]. Proteins of the peroxisomal lumen contain either a PTS1 [19] or a PTS2 signal [20]. The PTS1 signal is a specific tripeptide located at the C-terminal end of the protein, and is recognized by the Pex 5 receptor, a translocator for PTS1 containing proteins [21]. The ER origin of peroxisomes, combined with their capacity to import completely folded proteins, would render them ideally suited for secretion of intracellular proteins.
To enable this we have decorated Aspergillus niger peroxisomes with the A. niger ortholog of the v-SNARE Snc1 (SncA), by expressing it as a chimera with the A. niger ortholog of the peroxisomal membrane protein Pmp22 (PmpA) [22]. In Figure 1 panel $\mathrm{C}$ a schematic representation of the fusion of peroxisomes with the plasmamebrane is shown. The modified peroxisomes were able to fuse with the plasma membrane as evidenced by electron microscopy and extracellular secretion of peroxisomal accumulated proteins, which were tagged with the PTS1signal peptide -SKL. We have named this novel technology peroxicretion, for peroxisome-mediated intracellular protein secretion.

\section{Results}

\section{PTS I mediated peroxisomal import in A. niger}

The PTS1 receptor Pex5 is responsible for recognition and transport of PTS1-containing proteins into peroxisomes [21]. To confirm that PTS1 signals will result in peroxisomal localization in A. niger we have identified a Pex5 ortholog in the genome of A. niger (Genbank 4989140). Amino acids important for PTS1 recognition are conserved in the Pex5 ortholog (figure 1), suggesting that the presence of an -SKL sequence at the C-terminus of model proteins will lead to peroxisomal localization. Indeed, expressing -SKL tagged eGFP in A. niger (figure 2) caused a punctated pattern typical for peroxisomal localization. This result indicated that -SKL mediated peroxisomal targeting occurs in A. niger as expected based on the presence of a Pex 5 receptor ortholog.

\section{Decoration of A. niger peroxisomes with v-SNARE molecules}

To enable fusion of peroxisomes with the plasma membrane it was necessary to identify a peroxisomal membrane anchor, which could be used to place the v-SNARE SncA on the peroxisome. This peroxisomal membrane anchor should have the N-terminus positioned towards the cytosol, enabling $\mathrm{N}$-terminal fusions. The resulting chimeric protein is anchored in the peroxiomal membrane with the N-terminal fused SncA positioned at the cytosolic side of the peroxisome. Using the CBS prediction server http://www.cbs.dtu.dk/services/ we predicted the topology of the A. niger ortholog of Pmp22, which has been studied in Arabidopsis and in mammalian cells $[22,23]$. A membrane topology was predicted of 4 TMD's with the $\mathrm{N}$-terminus positioned at the cytosolic side of the peroxisome. PmpA contains two peroxisomal targeting regions with similar clusters of basic amino acids, interacting with Pex19p [22]. This prediction is in agreement with experimental evidence determining the topology of Pmp22 in Arabidopsis and mammalian cells [22,23]. To determine whether the Pmp22p ortholog of A. niger localises to the peroxisomes we constructed an eGFP-pmpA chimera and expressed this fusion gene in A. niger. We 

K. lactis
S. cerevisiae
A. niger -- MDVGS CSVGNNPLA QLHKH TQQNKS LQF N-QKNNGRLNESP-LQGTNKP G ISEAFIS
MSF LGGAECS TAGNP LTQFTKHVQDD KSLQRDRLVGRGP GGMQEGMRS RGMMGGQD QMMD

QVGYQN-PASNVAQNF KTHVNEVSNANRF QMD QFMNRSPGF S---DGQLGMAPVP SAILS NVNA I SQENMANMQRF INGEP L I DDKRRMEI GP SS GRLPP F SNVH S LQTSANP TQ I KGVN EF AQQPGQ IP GAP P QP F AMEQLRRELDQF QT TP PRTGSPGWAAEF DAGEHARMEAAFAGP $\therefore \quad$ : $:::$ : $\quad$ : : .

HGP RF GLK--KQD S GS SNMSA GD TAQHSRSWGNEFNSRSP QQ GLASRVNNVER ISNTNSM DI S HWSQE--FQGSNS I QNRNAD T GN SEKAWQRGS T TASSRF Q YP NTMMNNYA YA.SMNS L QGP MMNNA S GFTP AEF ARF QQ QSRAGMP QTANHVA SAP SP MMAGYQRPMGMGGYMGMGGM . . . . . . $\quad$. : . . . * . . . .

SS YRP GMSR I GRPMMHTG I SS LHN------YS HMS QQTPQMS SDD GVLADKQWNEQFEAL SGSRLQSP AFMNQQQS GRS KEGVN------EQEQQPWTDQFEKLEKEVSENLD INDE IEK GMMP $Q$ TF NP MAMQQQP AEATT QDKGKGRMVELDDENWEAQF AEMETAD TQKLDDEANAAV

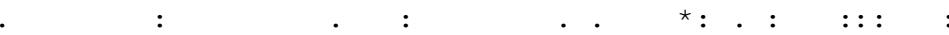

EKAVAENLTMEDNKEETKEEIVVEDGYQADFQEVWD---KLQAETADNNLET SDS-QWEK EENVSEVEQNKP ETVE-KEEGVYGDQYQSDF QEVWDS I HKDAEEVLP SELVNDDL-NLGE EAELNDLDRSVP QD SGD SAFESVWQRVQAETATNRKLAEGETDFN ID DNL HMGEMGEWD G

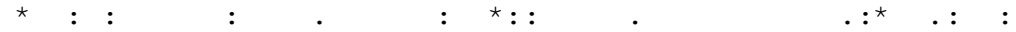

DYARYMTGKA TH I P PYRF DNDNOYMHNPNAYE I GC I LMENGA KLSEAALAFEAAVOEDPA DYLKY LGGRVNGN IEYAF QSNNEYFNNP NAYK I GC LLMENGAKLSEAALAFEAAVKEKPD FDTLNTRF RNPQLGDYMFEEDNVFRSVSNPFEEGVK IMREGGNLS LAALAFEAAVQKDPQ

$$
: \quad \star \star: .{ }^{\star}: \quad{ }^{\star} .::{ }^{\star} \quad{ }^{\star} .:^{\star} .: \star \star \star \star \star \star \star \star \star \star \star \star ~::{ }^{\star}
$$

HVDAWLKLGLVQT QNEKEMNG I SALE QCLSLDP TNQQALMT I S ISY I NEGYDL TAF SMLN HVDAWLRLGLVQTQNEKE LNG I SALEECLKLDP KNLEAMKTLA ISY INEGYDMSAF TMLD HVQAWTMLGSAQAQNEKELPA IRALEQALKIDANNLDALMGLAVSYTNEGYDSTSYRTLE

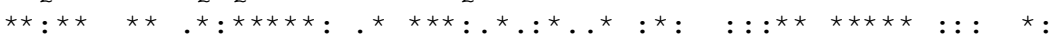

RWLD SKYPELT--RSP T ID-----EANIDRF NLSKQVI TKY LQVANALPQVDP EVQLGLG KWAE TKYP E IWS -RIKQQDDKF QKEKGF THI DMNAH I TKQF LQLANNL ST I DP EI QLCLG RWLSVKYP Q I INP NDVS SEAD LGF TDRQLLHDRVTDLF IQAAQLSP S GEQMDP DVQVGLG

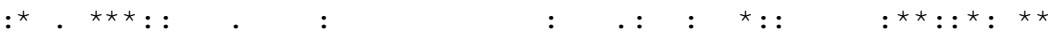

TLFYANEEFGKT IDCERTALEVNP----NDE----LMWNRLGASLANSNRSEEAIQAYHK LLFYTKDDF DKT IDCF ESALRVNP----NDE----LMWNRLGASLANSNRSEEAIQAYHR VLFYCAEEYDKAVDCF SAALASTESGT SNQQEQLHLLWNRLGATLANSGRSEEAIEAYEQ

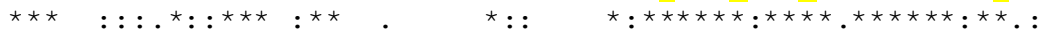

ALALKPSFVRARYNLA I S SMN I GCYKEAAES LLSALSMHEVEN----------VP I TGSV ALQLKPSFVRARYNLAVSSMNI GCFKEAAGYLLSVLSMHEVNT----------NNKKGDV ALNINPNFVRARYNLGVSC IN I GCYP EAAQHLLGALSMHRVVEQEGRERARE IVGGEGG I

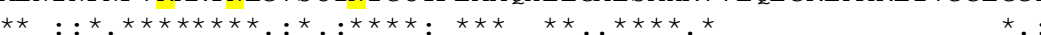

----------- VQSNN I LET LKRSFVAMDRRDLLEKVMP GMDLQQF RNEFNF GS-------LLNTYND TVIET LKRVF IAMNRDDLLQEVKP GMDLKRF KGEF SF DDEQLDRMI HVSONQS TNLYDT LRRVF SOMGRRDLADQVVAGMDVNVFRREFEF

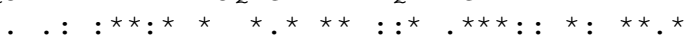

\section{Figure I}

Alignment of the PEX5 orthologs of S. cerevisiae, A. niger and $K$. lactis. Identical aminoacids are indicated with *, highly similar aminoacids are indicated with :, similar aminoacids are indicated with $\bullet$. The conserved aminoacids which are important for PTSI recognition are colored in yellow. 


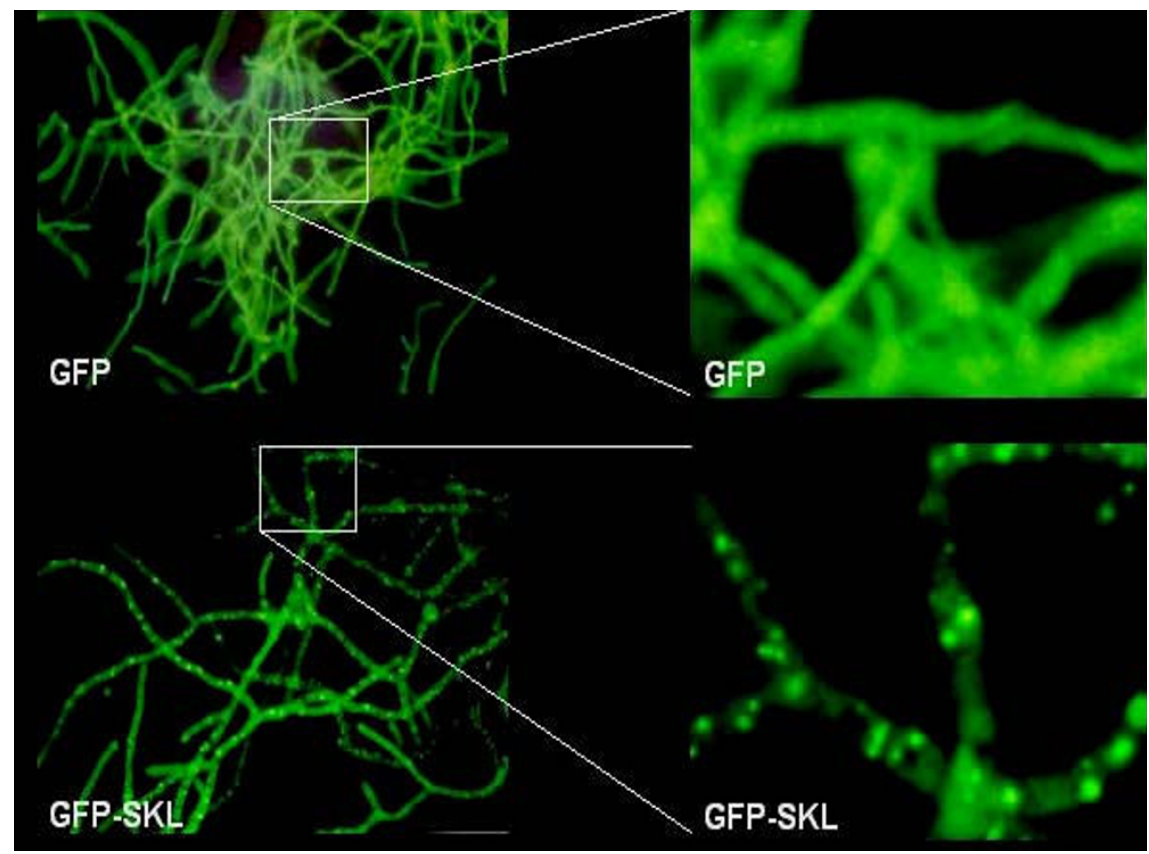

Figure 2

Peroxisomal localisation of eGFP-SKL. Fluorescence microscopy was applied to determine the sub cellular localisation of eGFP-SKL. As shown in the right panel the SKL tagged eGFP is found in a punctuated pattern whereas eGFP without SKL is localised throughout the cytosol as shown in the left part of the panel.

determined the localization of the eGFP-PmpA fusion protein by fluorescence microscopy. A similar punctated pattern was observed as with the eGFP-SKL fusion construct (figure 3), indicating that PmpA indeed can be used as peroxisomal membrane anchor. Moreover the majority of the eGFP-PmpA fusion protein is localised in peroxisomes. Since the SncA-PmpA fusion construct expressed using the same GlaA promoter in identical expression cassettes, the majority of SncA-PmpA will be localised to peroxisomes as well, this is shown in figure 4). The v-SNARE Snc1 in yeast, is intimately involved in fusion of Golgi derived vesicles to the plasma membrane. We have identified an Snc1 ortholog in A. niger (sncA), and fused the

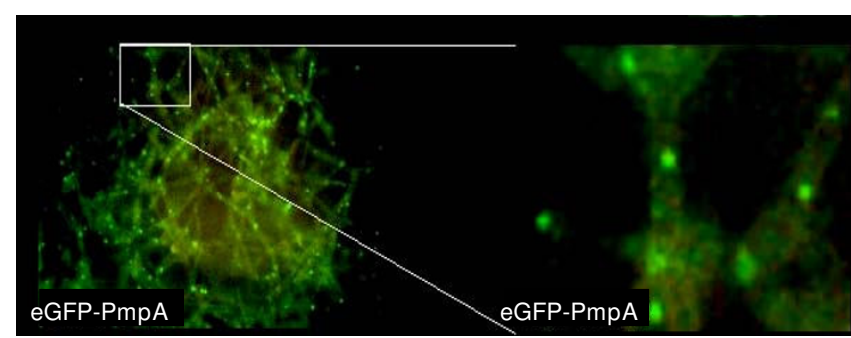

Figure 3

Peroxisomal localisation of eGFP-PmpA. The eGFP tagged PmpA showed a similar localisation pattern compared to eGFP-SKL (figure 2), indicating that eGFP-PmpA also localises to peroxisomes. gene (without its transmembrane coding region) to $p m p A$. When GFP-SKL and SncA-PmpA are co-overexpressed, a clustering of peroxisomes is observed (figure 5). This clustering is not observed when GFP-SKL is overexpressed (figure 2). This indicates that the SncA part exposed to the cytosol is interacting with itself thereby causing perxosimes to cluster. This behaviour of v-SNAREs has been observed before [24] and could be enhanced when overexpressed.

Using co-immunoprecipitaion we were able to identify the A. niger homologue of fox $2 p$ as a partner of the SncAPmpA fusion protein (figure 6). Fox2p is a peroxisomal protein involved in beta oxidation [25]. This is in line with the data presented which indicates the peroxisomal localisation of SNCA/PMPA.

\section{Peroxicretion in A. niger}

We cultivated A. niger co-expressing an SncA-PmpA fusion protein and an SKL-tagged eGFP in MEAM cultures as described in the materials and methods. After 24 hours, only transformants containing SncA-PmpA secreted eGFP-SKL into the extracellular medium, as evidenced by Western blot analysis using anti GFP antibody (figure 7). Expression of $s n c A-p m p A$ does not result in enhanced levels of extracellular acetamidase activity (used as selection marker), indicating that cell lysis is only limited. However the peroxicretion efficiency was also determined and in 


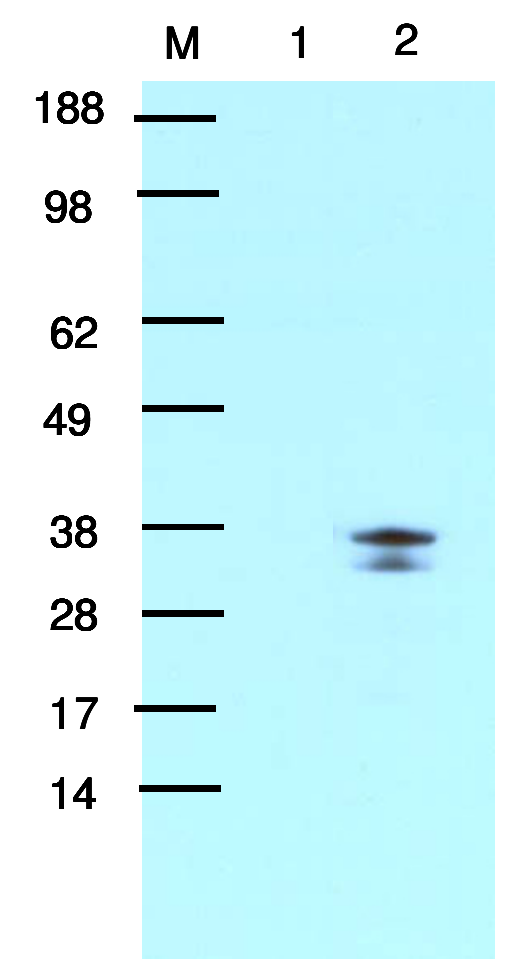

Figure 4

Expression of the fusion peptide SncA-PmpA. Cell free extracts were obtained from untransformed $A$. niger (lane I) and from SncA-PmpA transformed A. niger (lane 2). The Cell free extracts were subjected to SDS-PAGE and western blotting according the manufacturers instructions (Invitrogen) and detection was performed using a custom made antibody against SncA protein (Eurogentec). The expected size of the SncA-PmpA fusion protein is $35 \mathrm{kDa}$.

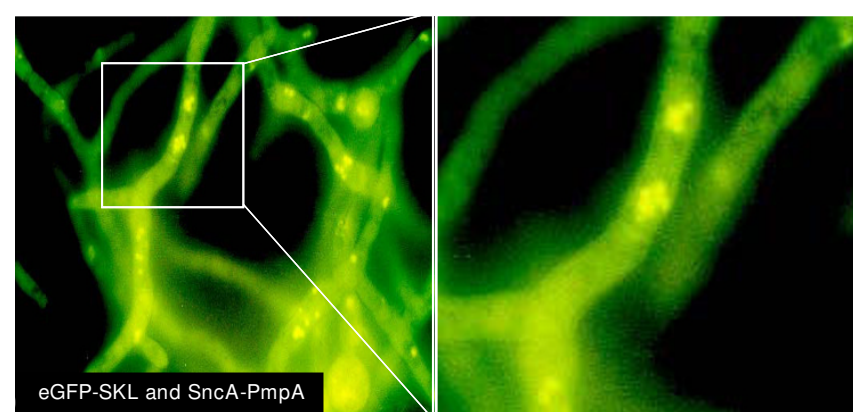

Figure 5

Co-expression of eGFP-SKL and SncA-PmpA in A. niger. $A$. niger transformants were grown on MEAM as described in materials and methods. After 48 hours biomass was transferred to glass slides and subjected to fluorescence microscopy. The decoration of peroxisomes with the $v$ SNARE SncA results in clustering of peroxisomes.

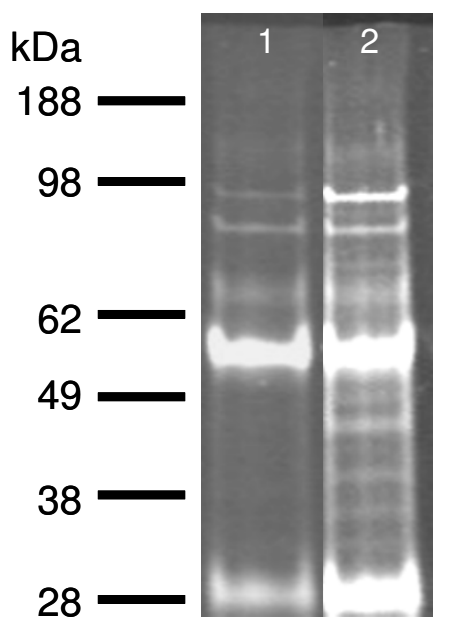

Figure 6

Analysis of co-immunoprecipitation complexes using anti-SncA by SDS-PAGE and Sypro ruby. SDS-PAGE (4-12\% Bis-Tris) gel loaded with the immunoprecipitation samples of wild type strain (lane I) and peroxicretion strain (wild type strain transformed with SncA-PmpA fusion construct) (lane 2) was stained with Sypro Ruby. The heavy and light chain of the used antibodies are running at $55 \mathrm{kDa}$ and $28 \mathrm{kDa}$. Proteins Differential bands were identified by MS/ MS.

the peroxicretion strain, overexpressing SncA-PmpA and eGFP-SKL, 55\% of the total GFP was extracellular. When we expressed only GFP-SKL we determined 25\% of the total GFP in the supernatant. This indicates that less than $50 \%$ of the extracellular GFP is due to lysis and more than $50 \%$ due to actual peroxicretion. We conclude that decoration of peroxisomes with the v-SNARE SncA resulted in fusion of peroxisomes with the plasma membrane, causing release of peroxisomal content in the extracellular medium. The applicability of this approach to secrete intracellular enzymes was further investigated using a set of enzymes indicated in figure 8 . We have expressed the indicated proteins in wild type A. niger (panel A) and in a peroxicreting $A$. niger (panel B), when indicated an SKL tag was placed at the $C$ terminus of the indicated proteins. The amylase proteins (amyA and amyB) are also visible, the presence of amyA is $\mathrm{pH}$ dependent. This explains why amyA is not always visible. The peroxicretion strain shows a slightly different acidification profile compared to the wild type strain. Results clearly showed peroxicretion of 3 overexpressed putative peroxisomal proteins from a SncAPmpA expressing strain (figure 8). Using MS/MS we could corroborate peroxicretion of at least one of those 3 proteins (strong similarity to catalase/peroxidase CpeB, (An01g01830)) and in addition identified one extra peroxicreted protein (similarity to endo-1,4-beta-xylanase XynD, (An11g03120)). The relatively low abundance of these proteins is likely to be caused by exposure to oxi- 


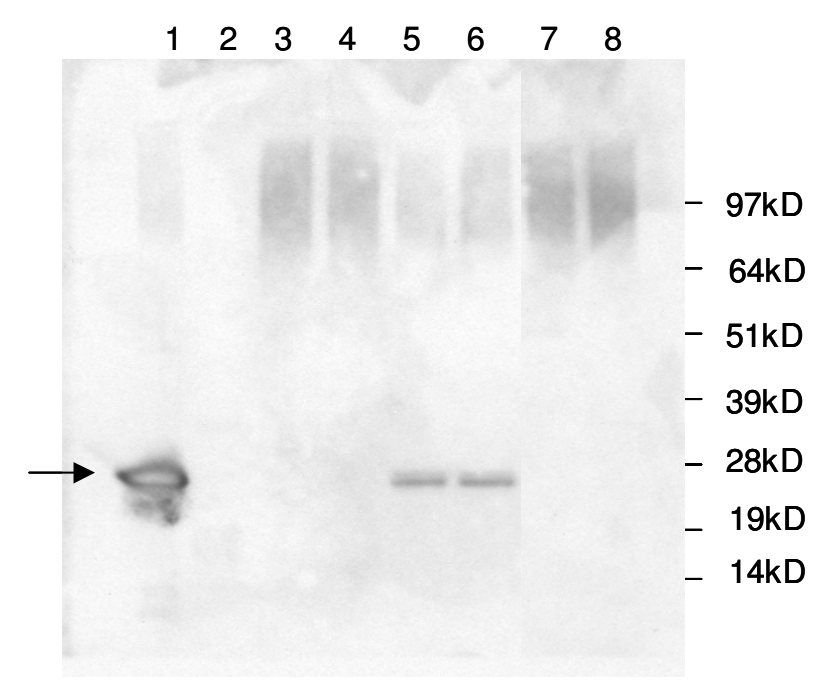

Figure 7

Secretion of eGFP-SKL mediated by SncA decorated peroxisomes. A. niger strains were cultivated for 24 hours in MEAM. 10 ul samples of the medium were taken and subjected to SDS-PAGE and subsequently to western blotting as indicated by the manufacturer (Invitrogen). Lane I: positive control eGFP, lane 2: MW marker indicated at the right hand side, lane 3 and 4: eGFP-SKL transformants, lane 5 and 6: eGFP-SKL, SNCA-PMPA co-transformants, lane 7 and 8: untransformed wild type strain.

dised conditions combined with the presence of extracellular proteases. It is evident that putative peroxisomal proteins can be peroxicreted as well as cytosolic proteins like the XynD orthologue. However the peroxisomal enzymes have a higher success rate probably because they are adapted to peroxisomal conditions in contrast to cytosolic proteins. Simple C-terminal SKL addition is sufficient to peroxicrete the XynD orthologue in SncA-PmpA expressing cells. Proteins which contain a putative PTS1 sequence like the catalase/peroxidase $\mathrm{CpeB}$ orthologue (An01g01830) and the alcohol oxidase orthologue (An18g05480) could be peroxicreted without modifications. The peroxicreted alcohol oxidase shows enzymatic activity in an $\mathrm{H}_{2} \mathrm{O}_{2}$ degrading assay, described in [26], and depicted in figure 9 . The wild type strain shows almost no $\mathrm{H}_{2} \mathrm{O}_{2}$ degrading activity in the supernatant whereas the peroxicretion strain shows an increasing in $\mathrm{H}_{2} \mathrm{O}_{2}$ degrading activity in the supernatant. This is most likely due to peroxicretion of endogenous catalases/peroxidases localised in peroxisomes.

\section{Finetuning of peroxicretion}

Fusion of peroxisomes with the plasma membrane was supported using electron microscopy. Inspection of ultrathin section of $\mathrm{KMnO}_{4}$-fixed cells revealed that peroxisomes were frequently located in close vicinity of the
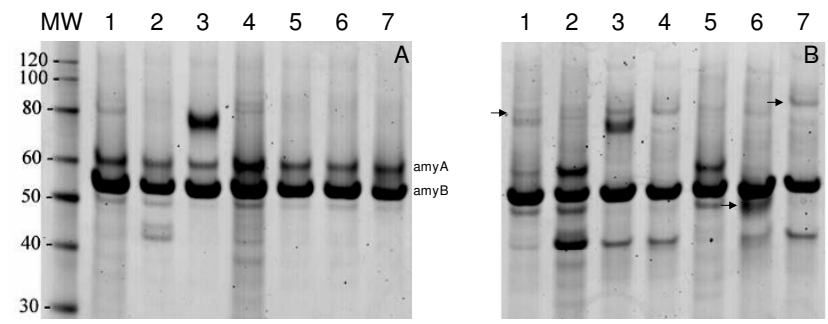

Figure 8

Peroxicretion of putative peroxisomal proteins. SDSPAGE analysis of shake-flask samples of the control strains (panel A) and peroxicreting strains (panel B), peroxicreting different intracellular $A$. niger enzymes as indicated. When indicated an SKL sequence was added to the $C$ terminus of the protein. After SDS-PAGE the cells were stained with coomassie brilliant blue. Endogenous amylases are indicated. The strains were grown for four days at $30^{\circ} \mathrm{C}, 250 \mathrm{rpm}$ In MEAM. Supernatant was isolated and loaded on a Nupage gel. Lane I: strong similarity to catalase/peroxidase CpeB, 84 kDa (An0 Ig0 I830), lane 2: strong similarity to chitinase I precursor Cts I, $48 \mathrm{kDa}(\mathrm{An02g07020)}$ an SKL sequence was added to the $C$ terminus of the protein, lane 3 : strong similarity to alpha-amylase precursor AmyA, $60 \mathrm{kDa}$

(An09g03 100) an SKL sequence was added to the $C$ terminus of the protein, lane 4: strong similarity to lipase LipP, $37 \mathrm{kDa}$ (An09g06390) an SKL sequence was added to the $C$ terminus of the protein, lane 5: similarity to endo- I,4-beta-xylanase XynD, 35 kDa (Anl Ig03 I 20) an SKL sequence was added to the $C$ terminus of the protein, lane 6: strong similarity to $D$ amino acid oxidase Daol, 4I kDa (An l4g05380) an SKL sequence was added to the $C$ terminus of the protein, lane 7: show strong similarity to several fungal alcohol oxidases, 74 kDa (An I8g05480).

cell membrane and often showed continuation with this membrane (figure 10A, B, C.). This was never observed in wild type cells without SncA-PmpA expression in which the organelles are scattered throughout the cytosol but are not seen in close proximity of the cell membrane (figure 10D). The efficiency of peroxicretion is likely to be controlled at the level of SNARE-pin formation during membrane fusion. In order to increase this efficiency of SNARE pin formation we have truncated the cytoplasmic tail of PmpA in order to place the v-SNARE SncA in closer proximity to the peroxisomal membrane. The peroxicretion efficiency is reduced when the N-terminus of PmpA is truncated with more than 18 amino acids, probably due to mislocalization (figure 11).

Another way of increasing the peroxicretion efficiency is to use $\mathrm{C} 2$ ceramide. Activation of CAPP by adding C2ceramide is known to result in increased availability of tSNARE, Sso1p, which is important for SNARE-pin formation $[26,27]$. Indeed, addition of C2-ceramide slightly enhanced the peroxicretion efficiency (figure 12). A third 
$\mathrm{H}_{2} \mathrm{O} 2$ degradation

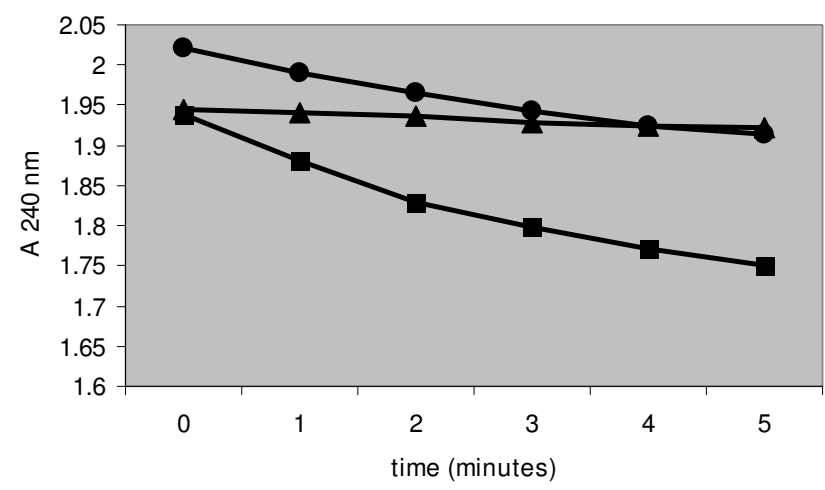

Figure 9

Enzymatic activity of peroxicreted alcohol oxidase. Using $100 \mu \mathrm{l}$ of supernatant of shakeflask grown $A$. niger cultures the degradation of $0.1 \% \mathrm{H}_{2} \mathrm{O}_{2}$ was monitored in $2 \mathrm{ml}$ milliQ by measuring absorption at $240 \mathrm{~nm}$. (black triangle) represents the $\mathrm{H}_{2} \mathrm{O}_{2}$ degrading activity of $\mathrm{I} 00 \mu \mathrm{l}$ supernatant of the wild type strain, (black circle) represents the $\mathrm{H}_{2} \mathrm{O}_{2}$ degrading activity of $\mathrm{I} 00 \mu \mathrm{l}$ supernatant of the peroxicretion strain and (black square) represents the $\mathrm{H}_{2} \mathrm{O}_{2}$ degrading activity of $100 \mu$ supernatant of the peroxicretion strain overexpressing gene ID 4990 I I 3 which shows strong similarity with several alcohol oxidases. approach to increase the efficiency of peroxicreion is to increase the number of peroxisomes. Overexpression of the A. niger ortholog of Pex11, which is known to be involved in peroxisomal proliferation [21] only resulted in minor peroxisomal proliferation in the A. niger transformants, while peroxicretion was not enhanced (figure 13). This may be explained by the fact that the increase in organelle numbers is not associated with a concomitant increase in matrix protein levels.

\section{Discussion}

In this study the v-SNARE SncA without its transmembrane domain was placed on the peroxisome using PmpA as a membrane anchor. The transmembrane domain of Snc1p is reported to be important for its function [28]. However, replacing the SncA-TMD by PmpA did not diminish the potential of SncA to enforce membrane fusion, since the peroxisomal content was released in the extracellular environment. PmpA as membrane anchor is sufficient for SncA to bring the membrane bilayers in close contact so that spontaneous membrane fusion occurs. We have selected peroxisomes because they can import completely folded proteins, which makes them ideally suited to transport and secrete proteins which are normally are localized intracellular. Recently, convincing evidence was presented that peroxisomes may originate from the ER [16]. This is important since the lipid composition of per-
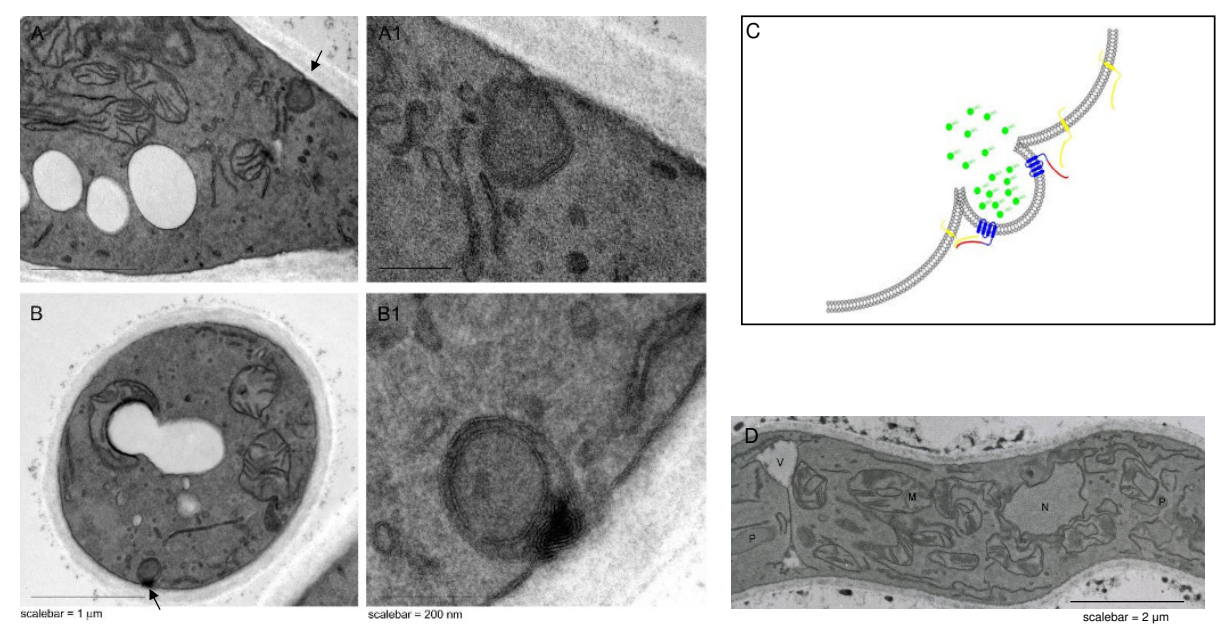

\section{Figure 10}

Survey of hyphal cells showing the position of a peroxisome (arrow) in the vicinity of the cell membrane in SncA-PmpA expressing A. niger. Panel A. Lengthwise overview of cellular membranes in SncA-PmpA expressing A. niger. Arrow indicates continuity of peroxisomes with the cell membrane (high magnification in panel Al). Panel B. Crosswise overview of cellular membranes in SncA-PmpA expressing A. niger. Arrow indicates continuity of peroxisomes with the cell membrane (high magnification in panel $\mathrm{BI}$ ). Panel $\mathrm{C}$. Schematic representation of the release of the peroxisomal content due to fusion of the peroxisome with the plasmamembrane. The target SNARE (Ssol ortholog) is shown in yellow as a transmembrane protein. The chimeric protein SncA-PmpA is depicted in blue (PmpA part) and red (SncA part). For simplicity Sec9 ortholog is not shown. The release of peroxisomal content is depicted, demonstrating peroxicretion of SKL tagged proteins (in green). Panel D. Detail of a glucose-grown A. niger WT cell, showing the presence of peroxisomes that are randomly scattered in the cytosol. M-mitochondrion, $\mathrm{N}$ - nucleus, $\mathrm{P}$ - peroxisomes, $\mathrm{V}$-vacuole. 


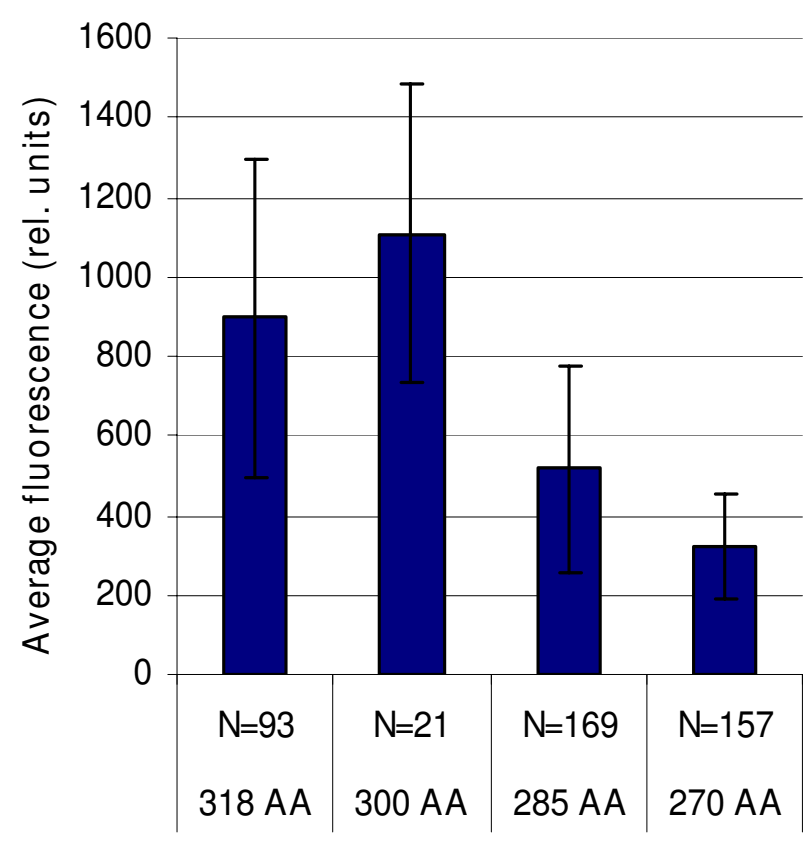

Figure I I

Mean fluorescence of eGFP-SKL overexpressing strains containing different truncated SncA-PmpA constructs. Fluorescence of individual samples was measured in supernatant of MTP cultures. SD is indicated as well as the number of transformants which were analysed.

oxisomes now is likely to be comparable to that of other ER/Golgi derived organelles like other secretory vesicles. Because of this similarity in lipid composition it appears unlikely that lipid incompatibility between peroxisomes and the plasma membrane would present a major hurdle for peroxicretion. Moreover, the small GTPase Rho1 is localized on peroxisomes through interaction with the peroxisomal membrane protein Pex25 [29]. Rho1p is known to play a role in actin reorganization and membrane dynamics. In yeast, Rho1 regulates polarized growth, and in the filamentous fungus A. niger polarized growth is even more predominant. In Arabidopsis, transportation of peroxisomes by actin filaments is reported [30] similar to transportation of secretory vesicles. This would be a possible mechanism how peroxisomes are able to be transported intracellular, Snc1 decorated peroxisomes are able to fuse with the plasmamembrane. The examples of Peroxicretion as described in this paper, shows that positioning of SncA on the peroxisomal membrane is sufficient for fusion of the peroxisome with the plasma membrane and subsequent release of its cargo. Interestingly, all these findings strengthen the notion that peroxisomes are derivatives of the secretory pathway. This renders peroxisomes as attractive vehicles for the transport of intracellular proteins towards the plasma membrane

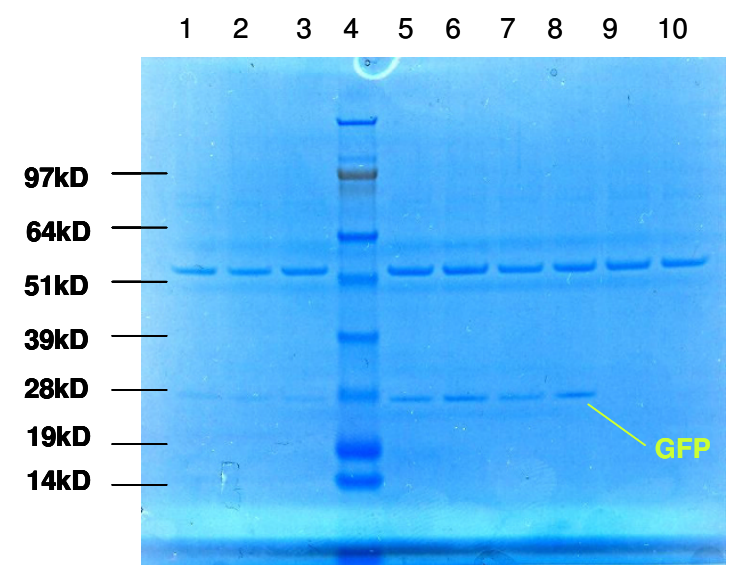

Figure 12

Ceramide stimulates peroxicretion, overexpression of PEXI I ortholog (An I I g02590) does not. I0 $\mu$ of supernatant after 48 hours growth of the strains transformed with the indicated constructs at $30^{\circ} \mathrm{C}, 250 \mathrm{rpm}$ in MEAM. Lane I; eGFP-SKL, lane 2; eGFP-SKL and C2 ceramide, lane 3; eGFP-SKL (duplo of lane I), lane 4; MW, lane 5; eGFP-SKL and SncA-PmpA, lane 6; eGFP-SKL, C2 ceramide and SNCAPMPA, lane 7; eGFP-SKL, SncA-PmpA and PEXII ortholog, lane 8; eGFP-SKL, C2 ceramide, SncA-PmpA and PEXI I ortholog lane 9; WT, lane I0; WT and C2 ceramide.

and secretion into the extracellular medium. It is however not excluded that peroxismes fuse to other intracellular compartments like endosomes before actual fusion with the plasmamebrane takes place. It is very likely that not all peroxisomes will be fused to the cellmembrane since the localization of PmpA fused proteins is not absolutely peroxisomal. In addition we do not observe decreased growth under conditions were peroxisomes are expected to be important. It is more likely that the peroxicretion concept as presented in this paper is not yet very efficient and that several key steps in the process like fusion of peroxisomes with the cell membrane, peroxisomal import of
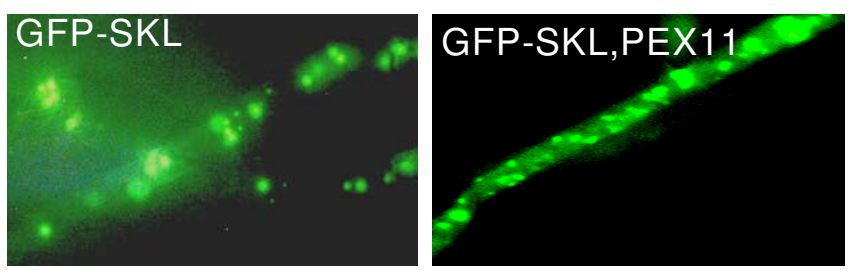

Figure 13

Overexpression of PEXI I ortholog leads to increased peroxisomal proliferation in A. niger. PEXI I ortholog overexpression leads to a minor increase in the number of peroxisomes however the peroxisomes seems to be smaller compared to the strain where only eGFP-SKL was expressed. 
proteins to be peroxicreted and stability of these proteins have to be improved.

\section{Conclusion}

This paper shows that it is possible to redirect intracellular trafficking of organelles by re-localizing v-SNARE molecules. The decoration of target organelles with selected vSNARE proteins is facilitated by using a modified membrane anchor which positions the v-SNARE onto the membrane of the target vesicle. This technology opens up numerous possibilities for studying intracellular vesicle trafficking in vivo. Besides this fundamental application the redirection of intracellular organelles also can be used in industrial biotechnology. As demonstrated in this paper, the targeting of proteins towards peroxisomes by using a C-terminal SKL sequence followed by fusion of modified peroxisomes with the plasmamembrane results in secretion of intracellular proteins. This process is designated peroxicretion. Although the levels of the peroxicreted products are still very low it shows the potential of the peroxicretion technology.

\section{Methods}

\section{Constructs, strains and transformation}

Standard molecular cloning techniques were performed. The constructs pGBFINSNP-2 to 5 contain the v-SNARE named sncA (An12g07570), fused to the N-terminal 318, 300, 285, or 275 amino acids of the peroxisomal membrane protein pmpA (An04g09130) respectively. The sncA-pmpA fusion gene is deposited as: GenBank DQ768214. Both genes were obtained by PCR on genomic DNA and the obtained nucleotide sequence was confirmed by sequencing (Baseclear, Leiden). The vector pGBFINGFS-1 contains eGFP with the amino acids SKL added to the C-terminus. The nucleotide sequence ( 5 '-TCCAAGCTC-3') encoding for the amino acids SKL was introduced at the C-terminus of eGFP by PCR. The construct pGBFINGFM-2 (GenBank DQ768213) was obtained by translational fusion of the eGFP and $p m p A$ ORF through PCR. The pGBFIN vector was also used for overexpression of different genes (An01g01830, An02g07020, An09g03100, An09g06390, An11g03120, An14g05380 and An18g05480). Using primers which were extended by 9 nucleotides encoding SKL the protein encoding sequences of An02g07020, An09g03100, An09g06390, An11g03120 and An14g05380 were modified at the $\mathrm{C}$ terminus. All of the above A. niger expression constructs are driven by the strong glucoamylase promoter and harbour flanking regions to ensure convenient targeting and expression in the fungal host as described previously [31]. Subsequent transformation of A. niger strain CBS 513.88 with the expression constructs was performed as previously described [32].

\section{Culture methods}

A. niger strains were inoculated at $5 \times 10^{6}$ spores $/ \mathrm{ml}$ MEAM consisting of: $6 \mathrm{~g} \mathrm{NaNO}_{3} ; 0.52 \mathrm{~g} \mathrm{KCl} ; 1.52 \mathrm{~g}$
$\mathrm{KH}_{2} \mathrm{PO}_{4} ; 1.12 \mathrm{ml} 4 \mathrm{M} \mathrm{KOH} ; 0.52 \mathrm{~g} \mathrm{MgSO}_{4} \cdot 7 \mathrm{H}_{2} \mathrm{O} ; 10 \mathrm{~g}$ glucose; 1 g casaminoacids; $22 \mathrm{mg} \mathrm{g} \mathrm{ZnSO}_{4} \cdot 7 \mathrm{H}_{2} \mathrm{O} ; 11 \mathrm{mg}$ $\mathrm{H}_{3} \mathrm{BO}_{3} ; 5 \mathrm{mg} \mathrm{FeSO} \mathrm{O}_{4} \cdot 7 \mathrm{H}_{2} \mathrm{O} ; 1.7 \mathrm{mg} \mathrm{CoCl} 2.6 \mathrm{H}_{2} \mathrm{O} ; 1.6 \mathrm{mg}$ $\mathrm{CuSO}_{4} .5 \mathrm{H}_{2} \mathrm{O} ; 1.5 \mathrm{mg} \mathrm{Na} \mathrm{MoO}_{4} \cdot 2 \mathrm{H}_{2} \mathrm{O} ; 50 \mathrm{mg}$ EDTA; 5 $\mathrm{mg} \mathrm{MnCl} .2 \mathrm{H}_{2} \mathrm{O} ; 2 \mathrm{mg}$ riboflavin; $2 \mathrm{mg}$ thiamin-HCl; 2 mg nicotinamid; $1 \mathrm{mg}$ pyrodoxin- $\mathrm{HCl} ; 0.2 \mathrm{mg}$ pantothenic acid; $4 \mu \mathrm{g}$ biotin;10 $\mathrm{ml}$ penicillin/streptomycine (Invitrogen) per liter. The strains were grown at $30^{\circ} \mathrm{C}$ and $250 \mathrm{rpm}$ for 1-3 days. When required conidiospores were obtained by growth on Potato Dextrose Agar (PDA, Oxoid, England) for 5 days at $30^{\circ} \mathrm{C}$, and isolated with MilliQ and a spatula.

\section{Western blot analysis}

SDS-PAGE was performed using NuPAGE Novex Bis-Tris precast gels (Invitrogen) according to the supplier's manual. Proteins were visualized by staining with SimplyBlue SafeStain (Invitrogen). Western analysis was performed with the XCell II semi-wet blotting module (Invitrogen) using MOPS buffer and nitrocellulose membrane $(0.45$ $\mu \mathrm{m}$ pore size) according to the supplier's manual. GFP was specifically detected by using 1:1,000 fold diluted GFP monoclonal antibody (Covance, California). After incubation with secondary antibody conjugated to horseradish peroxidase (anti-mouse, 1:1,000 dilution, PIERCE), immunoreactive proteins were detected by the enhanced chemiluminescence system (ECL, Amersham Pharmacia) and exposed to radiographic film (Kodak). The SNC 1 westerns were prepared similar to the procedure described above with the expection that the antiSNC1 antibody was custom made by Eurogentec.

\section{Immunoprecipitation of SncA-pmpA}

Frozen cells were disrupted in a mortar filled with liquid nitrogen and suspended in $0.5 \mathrm{ml} 20 \mathrm{mM}$ Sodium-Phosphate, $1 \%$ TritonX-100, 1 mM EDTA, and protease inhibiter. Cell lysate was voraciously vortexed and placed on ice for 10 minutes. TritonX-100 induces lyses of A. niger. The cell lysate was centrifuged for 5 minutes at $13.000 \mathrm{~g}$ and supernatant was taken. A pre-clearance step with $25 \mu \mathrm{l}$ was used to reduce the background of a-specific bonding to Protein-A-Sepharose (10\% Protein-A-Sepharose in 20 mM Sodium phosphate $\mathrm{pH}$ 7,4, 1 mM EDTA, 0,1\% Triton $\mathrm{X}-100)$. The soluble fraction was incubated with ProteinA-Sepharose and rotated head over head for 1 hour at $4{ }^{\circ} \mathrm{C}$. The supernatant was incubated with $2.5 \mu \mathrm{l} 10^{-5}$ diluted antibody SNC $\alpha$-SNC (serum $2^{\text {e booster }} \mathrm{NL}$ 03077, rabbit no $=\mathrm{SN} 1391$ ) for 1 hour at $4^{\circ} \mathrm{C}$ to bind the SNC for 1 hour at $4{ }^{\circ} \mathrm{C}$. In total $25 \mu$ l Protein-A-Sepharose was added to the sample to bind antibody SNC with bounded SNC and the incubation was extended for again one hour. Protein-A-Sepharose was used to facilitate spindown antibody SNC with bounded SNC. To remove the remaining antibody, Protein-A-Sepharose and the not bound proteins, the sample was washed once for $5 \mathrm{~min}$ - 
utes with 1,0 ml $20 \mathrm{mM}$ SodiumPhosphate, 0,1\% TritonX-100, $1 \mathrm{mM}$ EDTA, and protease inhibiter. The samples were treated with $25 \mu \mathrm{l}$ sample buffer and $5 \mu \mathrm{l}$ reducing agent. After heating the samples for 5 minutes at $95^{\circ} \mathrm{C}$ and centrifuging, $20 \mu \mathrm{l}$ supernatant was loaded on the 4\%-12\% SDS-PAGE gel. After electrophoresis, Sypro Ruby staining was performed. For 24 hours the gel was stained in $100 \mathrm{ml}$ Sypro Ruby staining. After staining, the gel was washed once with MilliQ. Instead of a Coomassie blue staining the gel was stained with a Sypro Ruby, because this is more sensitive. Sypro ruby does not need to be destained, because the dye does not bind irreversible to proteins and is therefore washable from the sample (Patton, 2000). The fragments were cut out of the gel and identified by mass-spectrometry.

\section{Microscopy}

For analysis of eGFP localization the fungal cells were grown at $30^{\circ} \mathrm{C}$ in MEAM for 1-2 days. Mycelium was transferred to microscope coverslides and studied under a Leica DMLA microscope connected to a CTRMIC unit. The apparatus was controlled by Qwin software from Leica. Electron microscopy was performed as described before [33].

\section{Digestion and LC-MSIMS analysis}

The sups were filtered over centrifugal devices (Pall) in tubes. Proteins with MW >100 kDa were filtered over 100 kDa centrifugal devices, proteins with MW 30-100 kDa were filtered over $30 \mathrm{kDa}$ centrifugal devices and proteins with MW 10-30 kDa were filtered over $10 \mathrm{kDa}$ centrifugal devices. $500 \mu \mathrm{L} \mathrm{MQ}$ was added on the filters and again the samples were centrifuged at $13000 \mathrm{rpm} 4^{\circ} \mathrm{C}$ for $15 \mathrm{~min}$ utes. $150 \mu \mathrm{L} 80 \mathrm{mM} \mathrm{NH}_{4} \mathrm{HCO}_{3}$ was added to the retentate of each of the samples after filtration and the retentate was transferred to $1.5 \mathrm{~mL}$ eppendorf tubes after pipetting up and down on the filter a couple of times. The proteins were denatured by incubation at $97^{\circ} \mathrm{C}$ for 10 minutes. $350 \mu \mathrm{L} 80 \mathrm{mM} \mathrm{NH}_{4} \mathrm{HCO}_{3}$ and $20 \mu \mathrm{L} 250 \mu \mathrm{g} / \mathrm{mL}$ trypsin were added and the proteins were digested by incubation at $37^{\circ} \mathrm{C}$ over night. $6 \mu \mathrm{L} 100 \mathrm{mM}$ DTT was added and the samples were incubated at room temperature for $30 \mathrm{~min}$ utes. LC-MS/MS was performed on the CapLC-QTOFII (Waters) system. For each of the samples a different MS/ MS method was made with the selected precursors for the over-expressed proteins. For each of the precursors theoretical fragmentation (MS/MS) spectra were made using Masslynx software (Waters) and the LC-MS/MS data was compared to these theoretical fragmentation spectra.

\section{Quantification of fluorescence}

Approximately $1 \times 10^{5}$ conidiospores were inoculated in $300 \mu \mathrm{l}$ MEAM (vitamins were omitted because of interference with the fluorescence measurements) per MTP well. After 5 days incubation at $30^{\circ} \mathrm{C}$ in an MTP (Nunc) the medium was separated from the mycelium and $200 \mu \mathrm{l}$ was transferred to a new MTP (Greiner, Fluotrac 200). Subsequently the fluorescence was measured on a Gemini spectra MAX (Molecular devices) controlled by SOFT max PRO v3.1.1 (Molecular Devices) using an excitation wavelength of $490 \mathrm{~nm}$ and an emission wavelength of $510 \mathrm{~nm}$. Additional settings: cut-off $495 \mathrm{~nm}$, PMT auto, calibrate on, 6 reads per well, 9 points per well. The amount of eGFP-SKL was determined in RFUs (relative fluorescent units).

\section{Abbreviations}

SNARE: Soluble Nsf-Attachment protein Receptors; ER: Endoplasmic Reticulum; PTS: peroxisomal Targeting Sequence; eGFP: enhanced Green Fluorescent Protein; TMD: Trans Membrane Domain; CAPP: Ceramide Activated Protein Phosphatase; MEAM: Minimal Enriched Aspergillus Medium.

\section{Authors' contributions}

The experiments were conceived and designed by CS, TW, $\mathrm{RD}$ and $\mathrm{PtH}$. MH and JdW performed the PmpA truncation studies and $\mathrm{PtH}$ performed the peroxicretion experiments. IM constructed the sncA-pmpA fusion gene which was used throughout this study. FL performed the peroxicretion experiments with endogenous proteins, which were designed by RD. RB performed electron microscopy. $\mathrm{MV}$ and IK interpreted the electron microscopy experiments. RD wrote the materials and methods section and submitted the sequences to Genbank. JM v/d L composed the peroxicretion protein test set. MA performed MS/MS experiments. All authors contributed to editing and writing of the paper.

\section{Acknowledgements}

We thank Rana Al-Majidi, Martine Steenbeek, Martine Spaans, Emmie Heeren, Brenda Vonk, Siebe Hartmans, Panagiotis Sarantinopoulos, Aldo Greeve, Lydia Schild and Ron Booy for experimental assistance. We specifically thank prof. Reinhard Jahn (Max Planck Institute, Goettingen) for scientific discussions.

\section{References}

I. Olkkonen VM, Stenmark H: Role of Rab GTPases in membrane traffic. Int Rev Cytol 1997, 176: I-85.

2. Wurmser AE, Emr SD: Phosphoinositide signaling and turnover: Ptdlns(3)P, a regulator of membrane traffic, is transported to the vacuole and degraded by a process that requires lumenal vacuolar hydrolase activities. EMBO J 1998 , 1 7:4930-4942.

3. Spang A: Vesicle transport: a close collaboration of Rabs and effectors. Curr Biol 2004, 14:R33-R34.

4. Rothman JE: Intracellular membrane fusion. Adv Second Messenger Phosphoprotein Res 1994, 29:8I-96.

5. Kabcenell AK, Goud B, Northup JK, Novick PJ: Binding and hydrolysis of guanine nucleotides by Sec4p, a yeast protein involved in the regulation of vesicular traffic. J Biol Chem 1990, 265:9366-9372.

6. TerBush DR, Maurice T, Roth D, Novick P: The Exocyst is a multiprotein complex required for exocytosis in Saccharomyces cerevisiae. EMBO J 1996, 15:6483-6494. 
7. Protopopov V, Govindan B, Novick P, Gerst JE: Homologs of the synaptobrevin/VAMP family of synaptic vesicle proteins function on the late secretory pathway in S. cerevisiae. Cell |993, 74:855-86|.

8. Katz L, Hanson PI, Heuser JE, Brennwald P: Genetic and morphological analyses reveal a critical interaction between the $C$ termini of two SNARE proteins and a parallel four helical arrangement for the exocytic SNARE complex. EMBO J 1998, 17:6200-6209.

9. Couve A, Gerst ]: Yeast Snc proteins complex with Sec9. Functional interactions between putative SNARE proteins. J Biol Chem 1994, 269(38):2339|-23394.

10. Rudolf R, Salm T, Rustom A, Gerdes HH: Dynamics of immature secretory granules: role of cytoskeletal elements during transport, cortical restriction, and F-actin-dependent tethering. Mol Biol Cell 200I, I 2:I353-1365.

II. Weber T, Zemelman BV, McNew JA, Westermann B, Gmachl M, Parlati F, Söllner TH, Rothman JE: SNAREpins: minimal machinery for membrane fusion. Cell 1998, 92(6):759-772.

12. Helenius A, Aebi M: Roles of $\mathbf{N}$-linked glycans in the endoplasmic reticulum. Annu Rev Biochem 2004, 73:1019-1049.

13. Spiro RG: Protein glycosylation: nature, distribution, enzymatic formation, and disease implications of glycopeptide bonds. Glycobiology 2002, I2(4):43R-56R.

14. Frand AR, Cuozzo JW, Kaiser CA: Pathways for protein disulphide bond formation. Trends Cell Biol 2000, 10:203-210.

15. Østergaard H, Tachibana C, Winther JR: Monitoring disulfide bond formation in the eukaryotic cytosol. J Cell Biol 2004 166:337-345.

16. Hoepfner D, Schildknegt DM, Braakman I, Philippsen P, Tabak HF: Contribution of the Endoplasmic reticulum to the peroxisome formation. Cell 2005, 1 22:85-95.

17. Kikuchi M, Hatano N, Yokota S, Shimozawa N, Imanaka T, Taniguchi $\mathrm{H}$ : Proteomic analysis of rat liver peroxisome: presence of peroxisome-specific isozyme of Lon protease. J Biol Chem 2004, 279:42I-428.

18. Walton PA, Hill PE, Subramani S: Import of stably folded proteins into peroxisomes. Mol Biol Cell 1995, 6:675-683.

19. Gould SG, Keller GA, Subramani S: Identification of a peroxisomal targeting signal at the carboxy terminus of firefly luciferase. J Cell Biol 1987, 105:2923-2931.

20. Swinkels BW, Gould SJ, Bodnar AG, Rachubinski RA, Subramani S: A novel, cleavable peroxisomal targeting signal at the aminoterminus of the rat 3-ketoacyl-CoA thiolase. EMBO J I99I, 10:3255-3262

21. Subramani S: Components involved in peroxisome import, biogenesis, proliferation, turnover and movement. Physiological reviews 1998, 78:17|-|84.

22. Brosius U, Dehmel T, Gartner J: Two different targeting signals direct human peroxisomal membrane protein 22 to peroxisomes. J Biol Chem 2002, 277:774-784.

23. Tugal HB, Pool M, Baker A: Arabidopsis 22-kilodalton peroxisomal membrane protein. Nucleotide sequence analysis and biochemical characterization. Plant Physiol 1999, I 20:309-20.

24. Wendler F, Tooze S: Syntaxin 6: the promiscuous behaviour of a SNARE protein. Traffic 200I, 2(9):606-6II.

25. Hiltunen JK, Wenzel B, Beyer A, Erdmann R, Fosså A, Kunau WH Peroxisomal multifunctional beta-oxidation protein of Saccharomyces cerevisiae. Molecular analysis of the fox 2 gene and gene product. J Biol Chem 1992, 267(10):6646-6653.

26. Beers RF Jr, Sizer IW: A spectrophotometric method for measuring the breakdown of hydrogen peroxide by catalase. J Biol Chem 1952, 195(I): 133-140.

27. Gurunathan S, Marash M, Weinberger A, Gerst JE: t-SNARE phosphorylation regulates endocytosis in yeast. Mol Biol Cell 2002, 13(5): 1594-1607.

28. Grote E, Baba M, Ohsumi Y, Novick Pl: Geranylgeranylated SNAREs Are Dominant Inhibitors of Membrane Fusion. J Cell Biol 2000, 151:453-466.

29. Marelli M, Smith Jl, Jung S, Yi E, Nesvizhskii Al, Christmas RH, Saleem RA, Tam YY, Fagarasanu A, Goodlett DR, Aebersold R, Rachubinski RA, Aitchison JD: Quantitative mass spectrometry reveals a role for the GTPase Rholp in actin organization on the peroxisome membrane. J Cell Biol 2004, 167:1099-III2.
30. Hashimoto K, Igarashi H, Mano S, Nishimura M, Shimmen T, Yokota E: Peroxisomal localization of a myosin XI isoform in Arabidopsis thaliana. Plant Cell Physiol 2005, 46:782-789.

31. van Dijck PW, Selten GC, Hempenius RA: On the safety of a new generation of DSM Aspergillus niger enzyme production strains. Regul Toxicol Pharmacol 2003, 38:27-35.

32. Kelly JM, Hynes MJ: Transformation of Aspergillus niger by the amdS gene of Aspergillus nidulans. EMBO J 1985, 4:475-479.

33. Waterham HR, Titorenko VI, Haima P, Cregg JM, Harder W, Veenhuis $M$ : The Hansenula polymorpha PERI gene is essential for peroxisome biogenesis and encodes a peroxisomal matrix protein with both carboxy- and amino-terminal targeting signals. J Cell Biol 1994, I 27(3):737-749.
Publish with Bio Med Central and every scientist can read your work free of charge

"BioMed Central will be the most significant development for disseminating the results of biomedical research in our lifetime. "

Sir Paul Nurse, Cancer Research UK

Your research papers will be:

- available free of charge to the entire biomedical community

- peer reviewed and published immediately upon acceptance

- cited in PubMed and archived on PubMed Central

- yours - you keep the copyright 